\title{
Transition to Adult Care in Youth with Epilepsy: One Center's Experience with a Transition Program and Its Integration within Telemedicine
}

\author{
Gina L. Jones ${ }^{1}$ Teresa Hickam ${ }^{2}$ Courtney Wellman ${ }^{3}$ Ann Modrcin ${ }^{4}$ Ahmed Abdelmoity ${ }^{1}$ \\ Jean-Baptiste Le Pichon ${ }^{1}$
}

${ }^{1}$ Department of Pediatric Neurology, Children's Mercy Hospital, Kansas City, Missouri, United States

2 Transition Program, Children's Mercy Hospital, Kansas City, Missouri, United States

${ }^{3}$ Neurology Clinic, Children's Mercy Hospital, Kansas City, Missouri, United States

${ }^{4}$ Department of Pediatric Rehabilitation, Children's Mercy Hospital, Kansas City, Missouri, United States

J Pediatr Epilepsy 2020;9:119-124.

Address for correspondence Gina L. Jones, DO, Department of
Pediatric Neurology, Children's Mercy Hospital, 2401 Gillham Road, Kansas City, MO 64108, United States (e-mail: gljones2@cmh.edu).

\begin{abstract}
Keywords

- transition

- telemedicine

- epilepsy

Neurology patients often have disorders that require life-long care and ongoing treatment. The transition of pediatric neurology patients to adult neurology care, particularly in epilepsy, is an evolving concept that is recognized as important for patients and families. Children's Mercy Hospital, Kansas City has an established transition program that has been integrated into the neurology clinic as a part of standard care. Our experience and utilization of a transition program has provided our patients with a unique opportunity, empowering them to navigate their own care and create a seamless transition to adult neurology. Through a collaboration with the American Academy of Pediatrics and the Department of Health Resources and Services Administration, we have proposed a practice model designed to create a medical home for children and youth with epilepsy in rural underserved areas of Kansas. One of the core end points of this study is transition of care for youth with epilepsy using telemedicine. In this article, we describe our experience with a transition program for epilepsy patients and describe the very early implementation of this program to a telemedicine transition program designed for underserved rural populations.
\end{abstract}

\section{Introduction}

The transition of pediatric neurology patients to adult neurology care, particularly in epilepsy, is an evolving concept that is recognized as important for patients and families. Likely causes for underutilization of transition programs are limited time and resources in a typical clinic environment. Due to the low numbers of child neurologists and pediatric epileptologists, children and young adults with chronic neurological conditions are frequently underserved. ${ }^{1}$ Bar-

received

July 26, 2020

accepted

August 6, 2020

published online

September 23, 2020 riers to care are even more apparent in rural areas due to distance from a specialist and economic burden. Telemedicine can bridge this gap by allowing for local specialty care and comanagement with the primary care provider, which in turn, decreases burden on the family and improves care. Transition can be implemented through telemedicine as standard of care and provide patients and families with the education and resources needed to direct their care and navigate the transition to adult neurology.
Copyright @ 2020 by Georg Thieme Verlag KG, Stuttgart · New York
DOI https://doi.org/ 10.1055/s-0040-1716826. ISSN 2146-457X. 
Adolescence is a critical time in life where there is growing independence and self-determination. ${ }^{2}$ This is a time to develop relationships outside of the family unit and begin to develop personal, emotional, and financial independence from parents. These major life changes are often complicated by the burden of chronic medical conditions. ${ }^{3}$ These are only a few of the numerous reasons that the development of a transition program is critical to the care of youth with epilepsy. Children's Mercy Hospital, Kansas City (CMKC) is a tertiary referral center with a Level 4 Epilepsy Center. The transition program was initially launched in 2014, and in 2018, we integrated the hospital's transition program model into our general neurology and epilepsy clinics as standard of care. This program has been received as a positive and empowering experience for both patients and their families and we are now expanding it, in the context of a Health Resources and Services Administration (HRSA) funded program, to reach out to underserved populations in rural regions of Kansas through telemedicine.

Evidence supports that a good transition of care program leads to improved outcomes, reduced health care costs, increased patient satisfaction, and improved quality of life. ${ }^{4}$ A systemic literature review on transition to adult care demonstrated two-thirds of the studies showed when a transition to care program is implemented, there is improved quality and service outcomes, adherence to care, improved quality of life and self-care skills, and less time between last pediatric and first adult visit. ${ }^{2}$ Thus, effective transition leads to improved outcomes with adherence to medication and follow-up care, whereas poor planning leads to risk of nonadherence to the medical plan and lack of follow-up care, which increases morbidity and mortality. ${ }^{3}$

Barriers to an effective transition are exacerbated in populations with poor access to specialized care. As it relates to pediatric epilepsy, access to specialists' care is limited by scarce resources, with only $\sim 2,000$ pediatric neurologists nationally, and few specialized Level 4 pediatric epilepsy centers in the United States. ${ }^{1}$ Most Level 4 pediatric epilepsy centers accredited by the National Association of Epilepsy Centers (NAEC) are in large cities, further limiting access for children and youth with epilepsy who live in rural areas (NAEC. www.naec-epilepsy.org). Limited access to these centers results in children and youth with epilepsy receiving inconsistent care across the United States, depending on the resources of a particular region. Underserved regions, including the Midwest and rural areas, suffer disproportionately from a lack of access to specialty epilepsy care. In these regions, the closest qualified epilepsy center is often not accessible due to distance and is frequently several hundred miles away. In addition to time constraints, the cost of travel and lodging are often prohibitive for families where the median household income is already low. This is true for Kansas, where $12.8 \%$ of the population lives below the Federal Poverty Level and 13\% of adults and 5\% of children are uninsured (American FactFinder, US Census, http://data. census.gov/cedsci/profile?g=0400000US20).

In this article, we make an argument for the importance of an effective transition of care from pediatric to adult neurology, especially as it relates to epilepsy. We describe our experience setting up a successful transition program at our center and we conclude with the description of a collaborative project with HRSA and the American Academy of Pediatrics (AAP), designed to facilitate transition to adult care via telemedicine for patients in underserved and rural areas of Kansas.

\section{Effective Transition Models}

Transition is a longitudinal and purposeful process in which a pediatric patient eventually transfers to adult care in an adult health care system. ${ }^{5}$ Transition to adult care involves more than a direct transfer of care and should begin early to prevent adverse outcomes. ${ }^{6}$ The transfer planning phase generally begins a few years before the targeted age of transfer from child to adult-oriented care (generally around 18-21 years of age). Ideally, the transition process should begin by introducing the policy and concepts of transition as soon as 12 to 14 years of age. Unfortunately, many patients report inadequate support and services when transitioning to adult care. ${ }^{7}$ In one study, it was reported that less than $50 \%$ of children with special health care needs had been assisted in transition by their pediatric provider. ${ }^{6}$ It is therefore essential that transition be a phased approach over time that includes psychosocial resources and resources to promote independence and selfadvocacy. ${ }^{4}$ It is also important to recognize that patients with complex medical needs often require more individualized planning and collaborative care. ${ }^{2}$

Having a health care transition plan creates a shared responsibility between pediatric and adult health care teams. The AAP, in partnership with Got Transition, developed a structured process that can be incorporated into any practice and customized to the patient to address medical complexity, social needs, and medical compliance. ${ }^{2}$ A transition program should be created with outreach to pediatric consultants for adult providers caring for patients with pediatric-onset disorders. The transition program should provide updated lists of community resources and adult providers that are willing to care for patients with pediatric-onset disorders or those with special needs. A team approach is often helpful and may include nurses, social workers, home health physicians, information technology, and insurance. There are multiple ways to develop an effective transition program, but most include a good patient summary from the pediatric neurologist and a phased transition to the adult neurologist. It is often felt a "joint" transition clinic visit with the pediatric neurologist is useful as it provides the adult care team with patient background and care while the family provides the psychosocial background. $^{3}$

\section{Transition in the Child Neurology Clinic}

The child neurology team serves as the primary agent in ensuring an appropriate transition plan exists and is routinely updated. The family should be provided a "transfer packet." This packet is used for care and coordination between providers. The neurologic care in the transition plan summarizes the patient's goals and care preferences, testing 
needs prior to transfer, the patient's understanding of their diagnosis, emergency plans, any advance plans of care, and guardianship for those with severe intellectual disabilities. ${ }^{8}$ It is important that child neurology providers foster relationships with their adult counterparts. The child neurologist, along with the young adult and caregiver, may then select an appropriate adult provider. When an adult provider is identified, a transition plan and medical summary should be given to the family and the adult provider. Once the patient attends the first appointment, it is documented in the child neurology medical record to confirm completed transition.

An assessment of expected competency to determine if power of attorney or legal guardianship is needed and should begin by 16 years of age. Our program recommends a referral to social work at this age to allow ample time for health care providers to make this assessment and the parent/caregiver to make the needed legal preparations.

\section{Special Considerations when Transitioning Children with Epilepsy}

Epilepsy is a complicated disease and transition should start early and be customized to the individual and their level of disability. A graduated approach is needed for a successful transition of care. Several steps of the graduated approach can and should be completed by team members other than the treating physician, including nurses and social workers who are an integral part of this process (see the following additional recommendations for transition in youth with epilepsy).

Additional recommendations to consider for transition in youth with epilepsy are as follows: ${ }^{9}$

- Identify barriers to successful transition (may include medication compliance, unplanned pregnancies, driving, coexisting psychiatric disorders, adult neurologists being uncomfortable with pediatric-onset or genetic epilepsies, or difficulty tailoring care to those with intellectual disabilities).

- Consider repeat epilepsy evaluation (may require repeat electroencephalogram studies, imaging, and genetic testing).

- Make sure that there is access to vagus nerve stimulation and dietary therapies (including ketogenic diet).

- Prepare the pediatric medical records needed for appropriate transition. This includes a detailed epilepsy history with previous evaluation and treatments, psychosocial assessments, current emergency care plan, goals of continued care, any needed additional referrals, and addressing legal or financial complications prior to transition of care.

It is essential to slowly initiate phased transition planning with education, and annual readiness questionnaires, encompassing current medications, medication side effects, genetic/reproductive counseling, driving, wellness, and the effects of drugs of abuse on their condition. ${ }^{8}$ Starting at 14 years of age, a comprehensive transition plan should be developed to meet the child's needs and the plan must be reviewed with the provider and updated with new goals annually.

\section{Example of a Successful Transition Program}

In 2017, the division of neurology at CMKC initiated a 1-year division wide quality improvement project designed to improve successful transition. A model of our hospital transition program was initially implemented in our Epilepsy Center and then pioneered for all patients in our general neurology clinics. Our clinic identified transition as a need, as patients and their families did not feel prepared and felt they lacked the knowledge for the plan to transition to adult neurology care. We started by training all the care team members (care assistants, nurses, social workers, and physicians in clinic) on the transition program. Previsit planning was implemented to identify follow-up patients, 15 years of age and older, for this program. During the intake process, the care team members invite the patient and family to connect to an online tool. The tool introduces the program by sharing our policy, showing a 2- to 3-minute video, directing the family to complete an electronic transition readiness assessment and selecting an annual goal. This automatically becomes a part of the medical record. During the visit, the provider reviews the patient's answers and annual goal, mutually agreeing to the goal, negotiating an alternative goal and/or adding additional goals. This goal is documented in the medical record. The process is repeated yearly until self-management goals are achieved and the adult provider has been identified. Once an appropriate transition to adult care has been completed, it is documented in the medical record.

A division goal was set following a measurement of the current rate of transition planning completion. After a 3month implementation phase, we moved into the maintenance phase. The last 3 months of data for that fiscal year showed a division average of $68 \%$ successful completion (14 advanced practice registered nurses, $25 \mathrm{MD} / \mathrm{DO}$ including fellows) with faculty averaging closer to $85 \%$ successful completion of the transition process (25 MD/DO including fellows). Our hospital transition program was implemented in all neurology clinics in the spring of 2018 and continues to this date (-Table $\mathbf{1}$ ). We have sustained the transition program throughout our entire neurology division, with positive feedback from patients. In fiscal year 2020, 388 patients were identified as eligible for the transition program and 357 had completed goal forms documented in the chart, which is a $92 \%$ sustainment. Patients and families continue to report adequate information regarding their treatment plan and getting their questions answered, related to transition planning, through surveys completed after each visit. This quality improvement work has demonstrated patients feel better prepared and are empowered to use what they have learned as they achieve their yearly transition program goals. Future goals include utilization of the hospital's newly created medical summary form which will 
122 Transition to Adult Care in Youth with Epilepsy Jones et al.

Table 1 Implementation and integration of the transition program into the Children's Mercy Hospital, Kansas City neurology clinics

\begin{tabular}{|l|l|l|}
\hline \multicolumn{2}{|l|}{$\begin{array}{l}\text { \% of eligible patients who completed } \\
\text { the transition steps for the entire division }\end{array}$} & $\begin{array}{l}\text { \% of eligible patients who completed } \\
\text { the transition steps for faculty only }\end{array}$ \\
\hline October-December 2017 baseline survey & \\
\hline October 2017 & $46 \%$ & \\
\hline November 2017 & $49 \%$ & \\
\hline December 2017 & $30 \%$ & $80 \%$ \\
\hline January-March 2018 implementation phase & $81 \%$ \\
\hline April-June 2018 assessment and integration phase & $88 \%$ \\
\hline April 2018 & $54 \%$ & \\
\hline May 2018 & $68 \%$ & $76 \%$ \\
\hline
\end{tabular}

document progress made and goals achieved through the transition years.

\section{Transition in the Age of Telemedicine}

Distance to the specialist, limited income, and care of other children can all make traveling to see the neurology specialist a real challenge for many families. These barriers to care became even more problematic during the coronavirus disease (COVID-19) pandemic. Yet, adverse effects associated with the lack of a structured health care transition program can result in difficulty with treatment plans, medication adherence, discontinuation of care, increased use of the emergency department for care, higher cost of care, and patient dissatisfaction. $^{2}$ The use of telemedicine paired with transition efforts can reduce these adverse outcomes by providing specialty care in these rural communities. This will subsequently reduce the burden of travel and expenses, promote communication and comanagement with the primary care provider, increase patient satisfaction, and ensure a smooth transition to adult epilepsy care. For this reason, it is essential that transition of care includes an initial joint appointment and/or pediatric neurology consultations. ${ }^{4}$ The use of telemedicine and tying it in with transition efforts will allow for local specialty care and decrease the burden of travel and strain on resources for the families of children with epilepsy. With the COVID-19 pandemic, CMKC has significantly increased the use of telemedicine, with 111 visits already having occurred via telemedicine, which is $37.5 \%$ of patient visits in 2020 thus far.

As in much of the United States, rural areas in Kansas represent a large proportion of the state and these areas often lack access to neurologists (-Fig. 1). Fortunately, telemedicine can serve as a bridge to provide epilepsy care

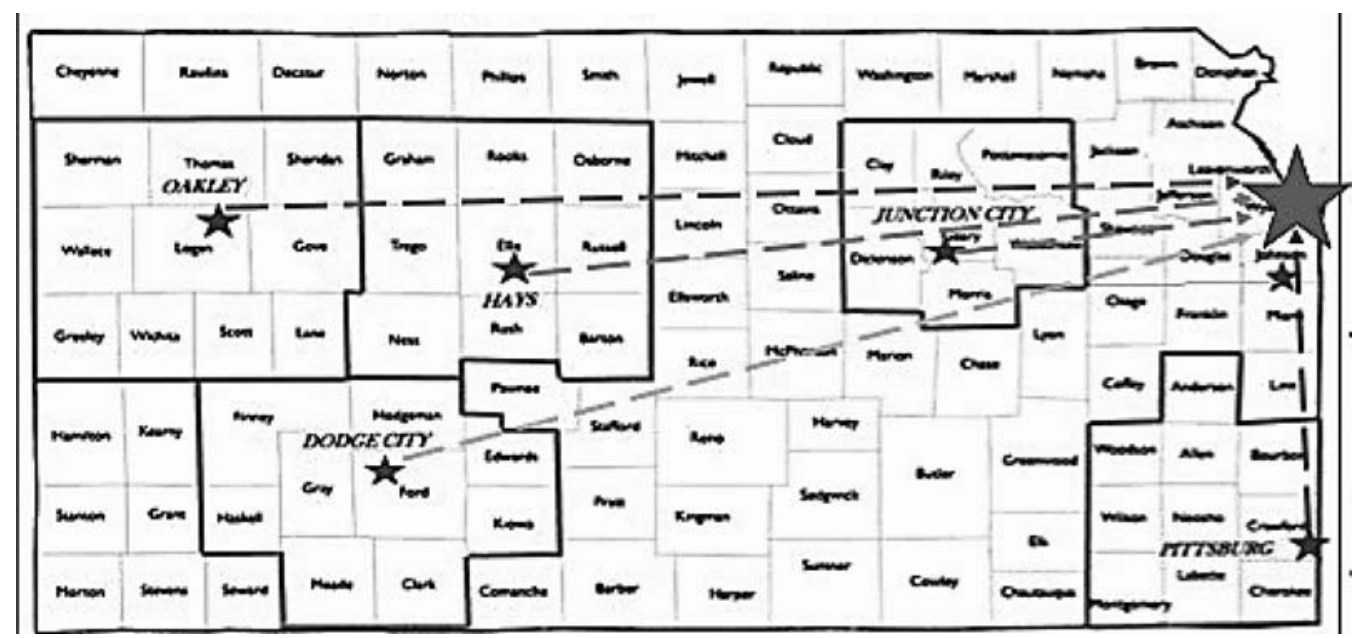

Fig. 1 Children's Mercy, Kansas City service areas for the HRSA grant. The distance from Oakley, KS to CMKC is 5 hours 4 minutes. The distance from Hays, KS to CMKC is 3 hours 48 minutes. The distance from Dodge City, KS to CMKC is 5 hours 14 minutes. The distance from Junction City, KS to CMKC is 1 hour 58 minutes. The distance from Pittsburg, KS to CMKC is 2 hours 1 minute. 


\section{Six Core Elements ${ }^{\mathrm{Tm}}$ Approach for Youth Transitioning to Adult Health Care}

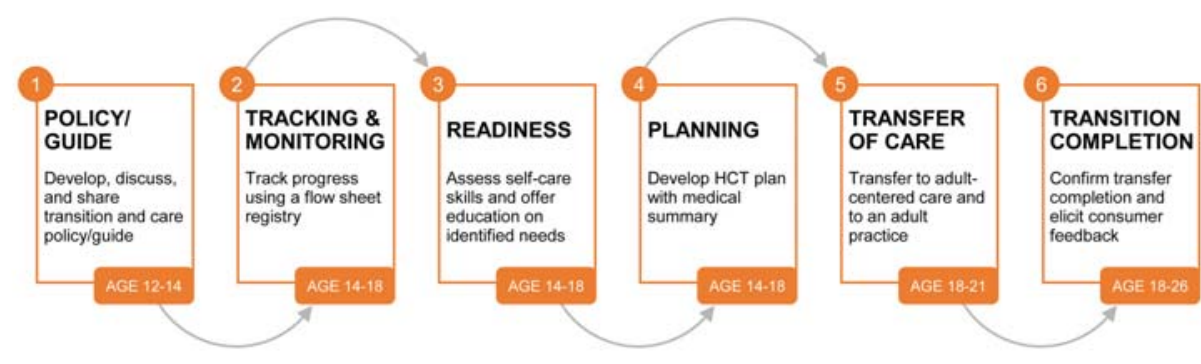

The Six Core Elements of Health Care Transition" ${ }^{14}$ are the copyright of Got Transition ${ }^{8}$. Non-commercial use is permitted, but requires attribution for any use, copy, or adaption.

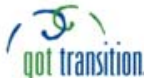

Fig. 2 Six Core Elements of Health Care Transition designed by Got Transition. (Reproduced with permission from Federally Funded Got Transition. Available at: https://www.gottransition.org/about/index.cfm.)

as these patients with complex disorders transition into adult care. CMKC has an established transition program that has been implemented into our clinic as standard of care (as described earlier). Through a collaboration with the AAP, we are now implementing a practice model designed to create a medical home for children and youth with epilepsy in rural underserved areas of Kansas. This is a 4-year project funded by the HRSA. The project is designed to increase access to quality coordinated health care in a model based on patient and family-centered medical homes for children and youth with epilepsy residing in rural and medically underserved areas in the state of Kansas. Challenges and considerations faced with the use of telemedicine in the rural setting include primary care providers with very few patients with epilepsy making complex care more difficult to coordinate, time constraints for providers in a busy rural primary care practice, family engagement, and technical issues. Keeping these considerations in mind, the objectives of this project include: improve access to care for children and youth with epilepsy, increase shared decision making by preparing families and primary care providers with education and skills, improve primary care provider and specialist communication and comanagement, provide effective epilepsy management education and skill development for primary care providers and families, and ensure a smooth transition to adult epilepsy care. This practice model incorporates the framework of the Six Core Elements of Health Care Transition designed by Got Transition (-Fig. 2) and key aspects of transition of care.

By providing care locally to those with epilepsy in rural settings, we have a unique opportunity to implement a transition program to these patients and their families and prepare them, over several years, for transition to adult neurology care. The hope is that through this program, the patients will be prepared with the information they need to navigate the multiple aspects of their neurologic care, allowing them to manage their own care and create a seamless transition to adult neurology. This grant is still in very early stages, but we are anticipating that our future data will show that by starting the transition process early (14 years old), and educating patients, families, and providers, a smooth transition can be achieved with minimal effort and result in better care (by breaking it down in small increments and goals), and needed information will be provided to navigate their health care as an adult. The CMKC and rural health care provider team members will be trained on the transition program process via telemedicine. Follow-up patients, 14 years of age and older, will be identified prior to their appointment. The process will then proceed as described earlier with a brief video, followed by a discussion with the physician and identification of an achievable goal each year. The process will then repeat each year until an appropriate adult provider has been identified and transition has been completed (-Fig. $\mathbf{3}$ ).

\section{Conclusion}

The transition of pediatric neurology patients to adult neurology care, particularly in epilepsy, is an evolving concept that is recognized as important for patients and families but is often underutilized given it requires time and resources. Epilepsy is a complex diagnosis and multiple barriers can prevent appropriate transition of care. With a structured program in place, a smooth transition to adult care can be achieved and provide patients with the ability to navigate care as an adult. Fortunately, for patients living in underserved areas, or during a pandemic as recently occurred with COVID-19, telemedicine can bridge the gap between specialty providers and primary care providers promoting a positive experience and appropriate transition to adult care. 
124 Transition to Adult Care in Youth with Epilepsy Jones et al.

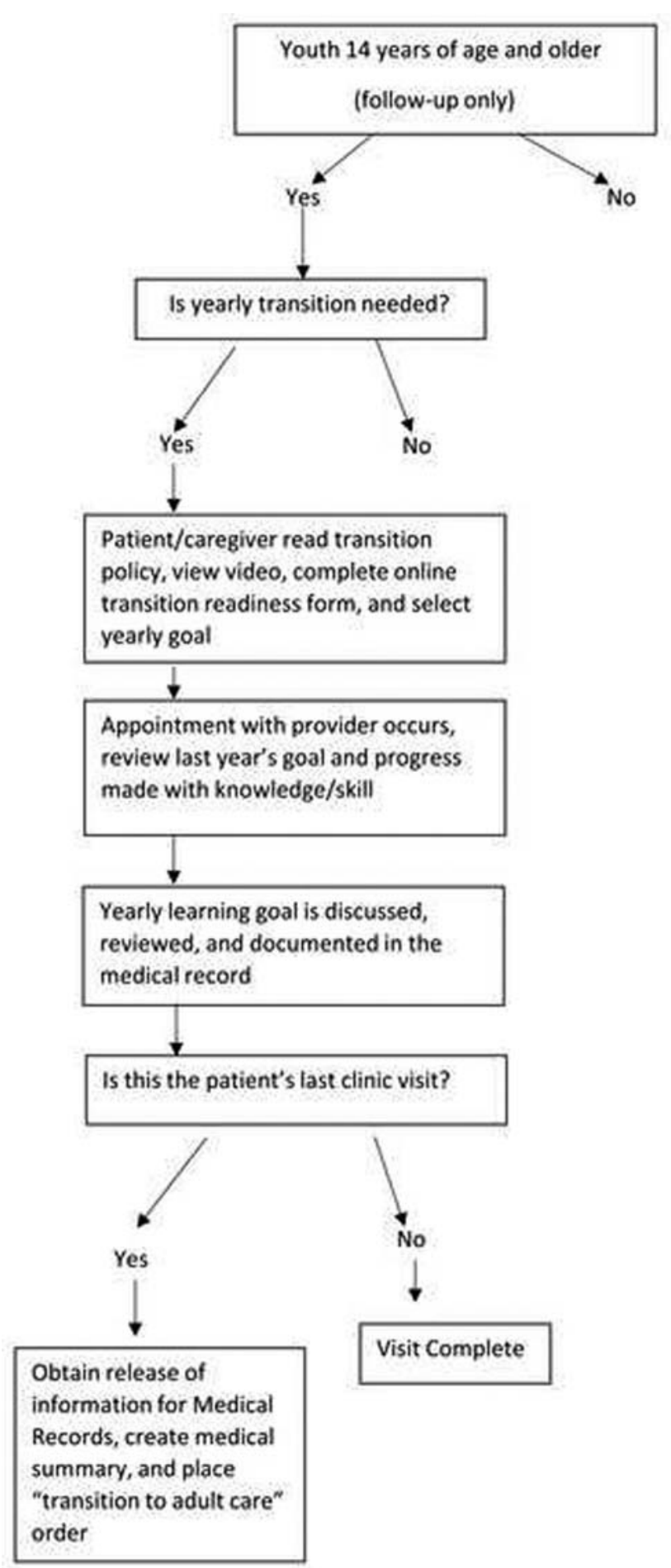

Conflict of Interest

None declared.

Acknowledgments

The Neurology Department at CMKC has been awarded a 4-year project funded by Health Resources and Services Administration (HRSA) to promote and create a medical home for youth with epilepsy in rural areas of Kansas. One of the core end points of this study is transition of care for children with epilepsy using telemedicine. We are grateful to Sarah Hueneke, MPH, for all her help in coordinating with the AAP and setting up the HRSA study.

\section{References}

1 Kang PB, Bale JF Jr, Mintz M, et al. Section on Neurology Executive Committee of the American Academy of Pediatrics, and the Board of Directors of the Child Neurology Society. The child neurology clinical workforce in 2015: report of the AAP/CNS joint taskforce. Neurology 2016;87(13):1384-1392

2 White PH, Cooley WC. Transitions Clinical Report Authoring Group; American Academy of Pediatrics; American Academy of Family Physicians; American College of Physicians. Supporting the health care transition from adolescence to adulthood in the medical home. Pediatrics 2018;142(05):e20182587

3 Rajendran S, Iyer A. Epilepsy: addressing the transition from pediatric to adult care. Adolesc Health Med Ther 2016;7:77-87

4 Brown LW. Medical transition from pediatric to adult care in neurology. Pract Neurol 2020;67:56-58

5 Rachas A, Lefeuvre D, Meyer L, et al. Evaluating continuity during transfer to adult care: a systematic review. Pediatrics 2016;138 (01):e20160256

6 Davis AM, Brown RF, Taylor JL, Epstein RA, McPheeters ML. Transition care for children with special health care needs. Pediatrics 2014;134 (05):900-908

7 Castillo C, Kitsos E. Transitions from pediatric to adult care. Glob Pediatr Health 2017;4:X17744946

8 Brown LW, Camfield P, Capers M, et al. The neurologist's role in supporting transition to adult health care: a consensus statement. Neurology 2016;87(08):835-840

9 Andrade DM, Bassett AS, Bercovici E, et al. Epilepsy: transition from pediatric to adult care. Recommendations of the Ontario epilepsy implementation task force. Epilepsia 2017;58(09): 1502-1517

Fig. 3 Children's Mercy, Kansas City Neurology Clinic flow chart for transition process in clinic and telemedicine. 